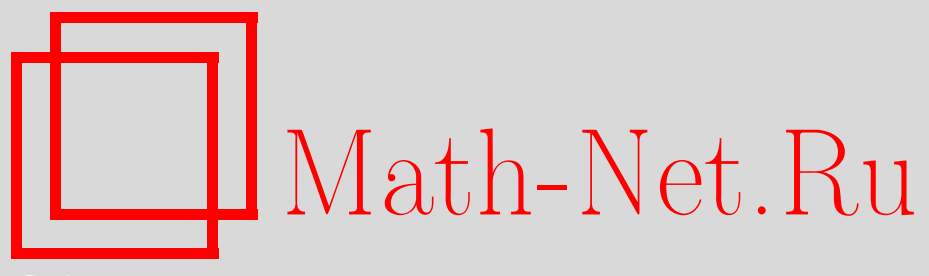

Д. В. Байдюк, Описание матриц рассеяния унитарных расширений изометрических операторов в пространстве Понтрягина, Матем. заметки, 2013, том 94, выпуск 6, 940-943

DOI: https://doi.org/10.4213/mzm10366

Использование Общероссийского математического портала Math-Net.Ru подразумевает, что вы прочитали и согласны с пользовательским соглашением http://www.mathnet.ru/rus/agreement

Параметры загрузки:

IP : 18.234 .156 .22

26 апреля 2023 г., 18:32:08

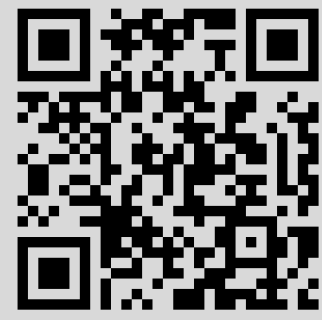




\section{Описание матриц рассеяния унитарных расширений изометрических операторов в пространстве Понтрягина}

\section{Д. В. Байдюк}

1. Введение. Многие задачи анализа, такие как проблема Неванлинны-Пика, проблема моментов и др., сводятся к исследованию некоторого симметрического оператора $A$ в гильбертовом пространстве $H$. При этом важную роль играет формула Крейна для описания $\mathfrak{L}$-резольвент, полученная в [1] для симметрического оператора $A$ с конечными индексами дефекта и распространенная в [2] на случай операторов с бесконечными индексами дефекта. Явная формула для $\mathfrak{L}$-резольвентной матрицы симметрического оператора $A$ в терминах граничных троек была установлена в [3] (для симметрических операторов в пространстве Понтрягина см. [4]).

Аналогом формулы Крейна для $\mathfrak{L}$-резольвент в случае изометрического оператора $V$ в гильбертовом пространстве является формула описания матриц рассеяния унитарных расширений оператора $V$, полученная Аровым и Гроссманом в [5]. Формула Арова-Гроссмана послужила основой для построения теории абстрактной интерполяции в работах Кацнельсона, Хейфеца и Юдицкого (см. [6]), включающей в себя большинство известных классических интерполяционных задач.

В работах Маламуда и Могилевского [7], [8] метод граничных операторов был распространен на случай изометрических операторов и применен к задаче описания обобщенных резольвент изометрического оператора $V$ в гильбертовом пространстве. В [9] этот метод получил развитие на случай изометрических операторов, действующих в пространствах Понтрягина $\Pi_{\kappa}$, и на его основе получено описание обобщенных резольвент изометрических операторов в пространствах $\Pi_{\kappa}$. В настоящей работе мы применяем эти результаты к описанию матриц рассеяния изометрического оператора $V$ в $\Pi_{\kappa}$ и находим явную формулу для $\mathfrak{L}$-резольвентной матрицы оператора $V$. В дальнейшем эта формула будет применена к описанию решений индефинитной абстрактной интерполяционной проблемы и, в частности, к таким задачам, как проблема Неванлинны-Пика и бикасательная интерполяционная проблема в обобщенных классах Шура.

2. Граничные тройки и функция Вейля. Пусть $(\mathcal{H},[\cdot, \cdot])$ - пространство Понт-

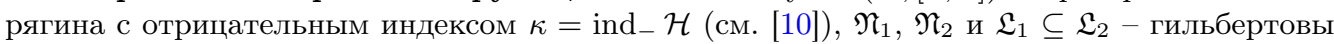
пространства, $\mathcal{B}\left(\mathfrak{N}_{2}, \mathfrak{N}_{1}\right)$ - множество ограниченных линейных операторов из $\mathfrak{N}_{2}$ в $\mathfrak{N}_{1}$. Пусть $V$ - изометрический оператор из $\mathcal{H} \oplus \mathfrak{L}_{2}$ в $\mathcal{H} \oplus \mathfrak{L}_{1}$ и $V^{[*]}-$ сопряженное линейное отношение из $\mathcal{H} \oplus \mathfrak{L}_{1}$ в $\mathcal{H} \oplus \mathfrak{L}_{2}$. Будем сокращенно писать $V^{-[*]}:=\left(V^{[*]}\right)^{-1}$. Заметим, что

$$
\operatorname{gr} V=\left\{\left[\begin{array}{c}
f \\
V f
\end{array}\right]: f \in \operatorname{dom} V\right\} \subset V^{-[*]} .
$$

Обозначим

$$
\mathbb{D}=\{\lambda \in \mathbb{C}:\|\lambda\|<1\}, \quad \mathbb{D}_{e}=\{\lambda \in \mathbb{C}:\|\lambda\|>1\}, \quad \mathbb{T}=\{\lambda:\|\lambda\|=1\},
$$

$S\left(\mathfrak{N}_{2}, \mathfrak{N}_{1}\right)$ - класс Шура сжимающих аналитических в $\mathbb{D}$ оператор-функций со значениями в $\mathcal{B}\left(\mathfrak{N}_{2}, \mathfrak{N}_{1}\right)$

ОПРеДЕЛЕниЕ 1. Совокупность $\Pi=\left\{\mathfrak{N}_{1} \oplus \mathfrak{N}_{2}, \Gamma_{1}, \Gamma_{2}\right\}$ назовем граничной тройкой изометрического оператора $V$, если

1) отображение $\Gamma=\left(\Gamma_{1}, \Gamma_{2}\right)^{T}: V^{-[*]} \rightarrow \mathfrak{N}_{1} \oplus \mathfrak{N}_{2}$ сюръективно;

DOI: $10.4213 / \mathrm{mzm} 10366$ 
2) справедливо обобщенное тождество Грина

$$
\left[f^{\prime}, g^{\prime}\right]_{\mathcal{H} \oplus \mathfrak{L}_{1}}-[f, g]_{\mathcal{H} \oplus \mathfrak{L}_{2}}=\left(\Gamma_{1} \widehat{f}, \Gamma_{1} \widehat{g}\right)_{\mathfrak{N}_{1}}-\left(\Gamma_{2} \widehat{f}, \Gamma_{2} \widehat{g}\right)_{\mathfrak{N}_{2}}
$$

для всех

$$
\widehat{f}=\left[\begin{array}{c}
f \\
f^{\prime}
\end{array}\right], \quad \widehat{g}=\left[\begin{array}{c}
g \\
g^{\prime}
\end{array}\right] \in V^{-[*]} .
$$

ЗАмечАниЕ 1. Если $\left\{\mathfrak{N}_{1} \oplus \mathfrak{N}_{2}, \Gamma_{1}, \Gamma_{2}\right\}$ - граничная тройка для изометрии $V$ и $\operatorname{dim} \mathfrak{N}_{1}=$ $\operatorname{dim} \mathfrak{L}_{1}, \operatorname{dim} \mathfrak{N}_{2}=\operatorname{dim} \mathfrak{L}_{2}$, то существуют унитарные операторы $X_{i}: \mathfrak{N}_{i} \rightarrow \mathfrak{L}_{i}, i=1,2$, такие, что совокупность $\left\{\mathfrak{L}_{1} \oplus \mathfrak{L}_{2}, X_{1} \Gamma_{1}, X_{2} \Gamma_{2}\right\}$ образует граничную тройку для $V$. В этом случае граничную тройку для $V$ можно выбрать так, что $\mathfrak{N}_{1}=\mathfrak{L}_{1}$ и $\mathfrak{N}_{2}=\mathfrak{L}_{2}$.

ПредЛОЖениЕ 1. Если $\iota: \mathcal{H} \oplus \mathfrak{L}_{1} \hookrightarrow \mathcal{H} \oplus \mathfrak{L}_{2}$ - оператор вложения $u \tilde{V} f:=\iota V f-$ изометрия из $\mathcal{H} \oplus \mathfrak{L}_{2}$ в $\mathcal{H} \oplus \mathfrak{L}_{2}$, то

$$
\widetilde{V}^{-[*]}=V^{-[*]}+\left\{\left[\begin{array}{l}
0 \\
u
\end{array}\right]: u \in \mathfrak{L}_{2} \ominus \mathfrak{L}_{1}\right\} .
$$

ПреДЛОЖЕНИЕ 2. Если $\left\{\mathfrak{N}_{1} \oplus \mathfrak{N}_{2}, \Gamma_{1}, \Gamma_{2}\right\}$ - граничная тройка для $V: \mathcal{H} \oplus \mathfrak{L}_{2} \rightarrow \mathcal{H} \oplus \mathfrak{L}_{1}$, то совокупность $\left\{\widetilde{\mathfrak{N}}_{1} \oplus \mathfrak{N}_{2}, \widetilde{\Gamma}_{1}, \widetilde{\Gamma}_{2}\right\}$, где $\widetilde{\mathfrak{N}}_{1}=\mathfrak{N}_{1} \oplus\left(\mathfrak{L}_{2} \ominus \mathfrak{L}_{1}\right)$,

$$
\widetilde{\Gamma}_{1}\left(\widehat{f}+\left[\begin{array}{l}
0 \\
u
\end{array}\right]\right)=\left[\begin{array}{c}
\Gamma_{1} \widehat{f} \\
u
\end{array}\right], \quad \widetilde{\Gamma}_{2}\left(\widehat{f}+\left[\begin{array}{l}
0 \\
u
\end{array}\right]\right)=\Gamma_{2} \widehat{f}, \quad \widehat{f} \in \widetilde{V}^{-[*]}, \quad u \in \mathfrak{L}_{2} \ominus \mathfrak{L}_{1},
$$

образует граничную тройку для $\widetilde{V}: \mathcal{H} \oplus \mathfrak{L}_{2} \rightarrow \mathcal{H} \oplus \mathfrak{L}_{2}$.

Для изометрического оператора $\widetilde{V}$ дефектные подпространства $\mathfrak{N}_{\lambda}(\widetilde{V})$ будем определять следующим образом:

$$
\mathfrak{N}_{\lambda}(\widetilde{V}):=\left\{f_{\lambda}:\left[\begin{array}{c}
f_{\lambda} \\
\lambda f_{\lambda}
\end{array}\right] \in \widetilde{V}^{-[*]}\right\} .
$$

В случае гильбертова пространства $\mathcal{H}$ понятие граничной тройки изометрического оператора $V$ введено в работе [7], а в случае пространства Понтрягина - в [11].

3. Теория представлений изометрических операторов. Пусть $P_{\mathcal{H}}$ и $P_{\mathfrak{L}_{1}}-$ это ортопроекторы на $\mathcal{H}$ и $\mathfrak{L}_{1}$, а $\widehat{\rho}\left(P_{\mathcal{H}} V\right)$ - это множество точек регулярного типа оператоpa $P_{\mathcal{H}} V$ (см. [13]).

ОПРЕДЕЛЕНИЕ 2. Будем писать, что $\lambda \in \rho\left(V, \mathfrak{L}_{2}\right)$, если $1 \in \widehat{\rho}\left(\lambda P_{\mathcal{H}} V\right)$ и

$$
\left(I-\lambda P_{\mathcal{H}} V\right) \operatorname{dom} V \dot{+} \mathfrak{L}_{2}=\mathcal{H} \oplus \mathfrak{L}_{2},
$$

$\lambda \in \rho\left(V^{-1}, \mathfrak{L}_{2}\right)$, если $1 \in \widehat{\rho}\left(\lambda P_{\mathcal{H}} V^{-1}\right)$ и

$$
\left(I-\lambda P_{\mathcal{H}} V^{-1}\right) \operatorname{ran} V \dot{+} \mathfrak{L}_{1}=\mathcal{H} \oplus \mathfrak{L}_{1} .
$$

Будем писать, что $\lambda \in \rho_{V}\left(\mathfrak{L}_{2}, \mathfrak{L}_{1}\right)$, если $\lambda \in \rho\left(V, \mathfrak{L}_{2}\right)$, а $\bar{\lambda} \in \rho\left(V^{-1}, \mathfrak{L}_{1}\right)$.

ОПредЕлЕниЕ 3 . При $\lambda \in \rho\left(V, \mathfrak{L}_{2}\right)$ обозначим $\mathcal{P}_{\mathfrak{L}_{2}}(\lambda)$ косой проектор на $\mathfrak{L}_{2}$ в разложении (3) и введем операторы

$$
\begin{aligned}
& \mathcal{Q}_{\mathfrak{L}_{2}}(\lambda):=P_{\mathfrak{L}_{2}} \widetilde{V}\left(I-\lambda P_{\mathcal{H}} V\right)^{-1}\left(I-\mathcal{P}_{\mathfrak{L}_{2}}(\lambda)\right): \mathcal{H} \oplus \mathfrak{L}_{2} \rightarrow \mathfrak{L}_{2}, \\
& \mathcal{Q}_{\mathfrak{L}_{1}}(\lambda):=P_{\mathfrak{L}_{1}} \mathcal{Q}_{\mathfrak{L}_{2}}(\lambda) .
\end{aligned}
$$

Определим также

$$
\widehat{\mathcal{P}}_{\mathfrak{L}_{2}}(\lambda)^{[*]}=\left[\begin{array}{c}
\mathcal{P}_{\mathfrak{L}_{2}}(\lambda)^{[*]} \\
\bar{\lambda}\left(\mathcal{P}_{\mathfrak{L}_{2}}(\lambda)^{[*]}-I_{\mathfrak{L}_{2}}\right)
\end{array}\right], \quad \widehat{\mathcal{Q}}_{\mathfrak{L}_{i}}(\lambda)^{[*]}=\left[\begin{array}{c}
\mathcal{Q}_{\mathfrak{L}_{i}}(\lambda)^{[*]} \\
\bar{\lambda} \mathcal{Q}_{\mathfrak{L}_{i}}(\lambda)^{[*]}+I_{\mathfrak{L}_{i}}
\end{array}\right], \quad \text { при } \quad i=1,2 .
$$

При этом

$$
\mathfrak{N}_{\bar{\lambda}}(\widetilde{V})=\left(\mathcal{P}_{\mathfrak{L}_{2}}(\lambda)^{[*]}+\bar{\lambda} \mathcal{Q}_{\mathfrak{L}_{2}}(\lambda)^{[*]}\right) \mathfrak{L}_{2} .
$$


Teорема 1. При $\lambda \in \rho_{V}\left(\mathfrak{L}_{2}, \mathfrak{L}_{1}\right)$ справедливы разложения

$$
\begin{aligned}
& V^{-[*]}=\operatorname{gr} V \dot{+} \widehat{\mathcal{P}}_{\mathfrak{L}_{2}}(\lambda)^{[*]} \mathfrak{L}_{2}+\mathcal{Q}_{\mathfrak{L}_{1}}(\lambda)^{[*]} \mathfrak{L}_{1} . \\
& \widetilde{V}^{-[*]}=\operatorname{gr} \widetilde{V} \dot{+} \widehat{\mathcal{P}}_{\mathfrak{L}_{2}}(\lambda)^{[*]} \mathfrak{L}_{2}+\mathcal{Q}_{\mathfrak{L}_{2}}(\lambda)^{[*]} \mathfrak{L}_{2} .
\end{aligned}
$$

При $\lambda \in \rho\left(V, \mathfrak{L}_{2}\right)$ определим операторы

$$
\begin{array}{rlrl}
\mathcal{G}(\lambda) & :=\left[\begin{array}{c}
\mathcal{Q}_{\mathfrak{L}_{1}}(\lambda) \\
I_{\mathfrak{L}_{2}}-\mathcal{P}_{\mathfrak{L}_{2}}(\lambda)
\end{array}\right], & \widetilde{\mathcal{G}}(\lambda):=\left[\begin{array}{c}
\mathcal{Q}_{\mathfrak{L}_{2}}(\lambda) \\
I_{\mathfrak{L}_{2}}-\mathcal{P}_{\mathfrak{L}_{2}}(\lambda)
\end{array}\right], \\
\mathcal{V}(\lambda):=\left[\begin{array}{c}
\widehat{\mathcal{Q}}_{\mathfrak{L}_{1}}(\lambda) \\
-\widehat{\mathcal{P}}_{\mathfrak{L}_{2}}(\lambda)
\end{array}\right], & \widetilde{\mathcal{V}}(\lambda):=\left[\begin{array}{c}
\widehat{\mathcal{Q}}_{\mathfrak{L}_{2}}(\lambda) \\
-\widehat{\mathcal{P}}_{\mathfrak{L}_{2}}(\lambda)
\end{array}\right] .
\end{array}
$$

Теорема 2. Пусть $\Pi=\left\{\mathfrak{N}_{1} \oplus \mathfrak{N}_{2}, \Gamma_{1}, \Gamma_{2}\right\}$ - граничная тройка для $V: \mathcal{H} \oplus \mathfrak{L}_{2} \rightarrow \mathcal{H} \oplus \mathfrak{L}_{1}$, a $\widetilde{\Pi}=\left\{\widetilde{\mathfrak{N}}_{1} \oplus \mathfrak{N}_{2}, \widetilde{\Gamma}_{1}, \widetilde{\Gamma}_{2}\right\}$ - граничная тройка для $\widetilde{V}=\iota V: \mathcal{H} \oplus \mathfrak{L}_{2} \rightarrow \mathcal{H} \oplus \mathfrak{L}_{2}$. Тогда для $\lambda, \mu \in \rho_{V}\left(\mathfrak{L}_{2}, \mathfrak{L}_{1}\right)$ оператор-функиии

$$
W(\lambda):=\left(\Gamma \mathcal{V}(\lambda)^{[*]}\right)^{*}, \quad \widetilde{W}(\lambda):=\left(\widetilde{\Gamma} \widetilde{\mathcal{V}}(\lambda)^{[*]}\right)^{*}
$$

удовлетворяют тождествам

$$
\begin{aligned}
& J_{\mathfrak{L}}-W(\lambda) J_{\mathfrak{N}} W(\mu)^{*}=(1-\lambda \bar{\mu}) \mathcal{G}(\lambda) \mathcal{G}(\mu)^{[*]}, \\
& J_{\widetilde{\mathfrak{L}}}-\widetilde{W}(\lambda) J_{\widetilde{\mathfrak{N}}} \widetilde{W}(\mu)^{*}=(1-\lambda \bar{\mu}) \widetilde{\mathcal{G}}(\lambda) \widetilde{\mathcal{G}}(\mu)^{[*]},
\end{aligned}
$$

где

$$
\begin{array}{ll}
J_{\mathfrak{L}}=\left[\begin{array}{cc}
I_{\mathfrak{L}_{1}} & 0 \\
0 & -I_{\mathfrak{L}_{2}}
\end{array}\right], & J_{\mathfrak{N}}=\left[\begin{array}{cc}
I_{\mathfrak{N}_{1}} & 0 \\
0 & -I_{\mathfrak{N}_{2}}
\end{array}\right], \\
J_{\widetilde{\mathfrak{L}}}=\left[\begin{array}{cc}
I_{\mathfrak{L}_{2}} & 0 \\
0 & -I_{\mathfrak{L}_{2}}
\end{array}\right], & J_{\widetilde{\mathfrak{N}}}=\left[\begin{array}{cc}
I_{\mathfrak{N}_{2}} & 0 \\
0 & -I_{\mathfrak{N}_{2}}
\end{array}\right] .
\end{array}
$$

ОпредЕлЕниЕ 4. Оператор-функции $W(\cdot)$ и $\widetilde{W}(\cdot)$, удовлетворяющие тождествам (11) и (12), называются резольвентными матрицами операторов $V$ и $\widetilde{V}$ соответственно.

Таким образом, $W(\cdot)$ и $\widetilde{W}(\cdot)$, определенные формулами $(10)$, являются резольвентными матрицами для операторов $V$ и $\widetilde{V}$ соответственно. При этом

$$
W(\cdot)=P_{\mathfrak{L}_{1} \oplus \mathfrak{L}_{2}} \widetilde{W}(\cdot) \uparrow \mathfrak{N}_{1} \oplus \mathfrak{N}_{2}
$$

ЗАмечание 2. Если в граничной тройке $\Pi=\left\{\mathfrak{N}_{1} \oplus \mathfrak{N}_{2}, \Gamma_{1}, \Gamma_{2}\right\}$ выполнено $\mathfrak{N}_{1}=\mathfrak{L}_{1}$, $\mathfrak{N}_{2}=\mathfrak{L}_{2}$ и $a \in \mathbb{T} \cap \rho_{V}\left(\mathfrak{L}_{2}, \mathfrak{L}_{1}\right)(\neq \varnothing)$, то, полагая $W(a)=I$, получаем из $(11)$

$$
W(\lambda)=I-(1-\lambda \bar{a}) \mathcal{G}(\lambda) \mathcal{G}(a)^{[*]} J_{\mathfrak{L}}, \quad \lambda \in \rho_{V}\left(\mathfrak{L}_{2}, \mathfrak{L}_{1}\right) .
$$

ОПредЕление 5 . Пусть $U: \widetilde{\mathcal{H}} \oplus \mathfrak{L}_{2} \rightarrow \widetilde{\mathcal{H}} \oplus \mathfrak{L}_{1}-$ унитарный оператор, являющийся расширением изометрического оператора $V: \mathcal{H} \oplus \mathfrak{L}_{2} \rightarrow \mathcal{H} \oplus \mathfrak{L}_{1}, \widetilde{\mathcal{H}}-$ пространство Понтрягина, содержащее $\mathcal{H}$, такое, что ind $\widetilde{\mathcal{H}}=$ ind $_{-} \mathcal{H}$. Оператор-функция $s(\lambda): \mathfrak{L}_{2} \rightarrow \mathfrak{L}_{1}$, определенная в $\mathbb{D} \backslash\left(\sigma_{p}(U) \cup \sigma_{p}\left(U P_{\mathcal{H}}\right)\right)^{-1}$ равенством

$$
s(\lambda)=P_{\mathfrak{L}_{1}}\left(I-\lambda U P_{\mathcal{H}}\right)^{-1} U \uparrow \mathfrak{L}_{2},
$$

называется матрищей рассеяния унитарного расширения $U$ изометрического оператора $V$ (см. [5], [11], [9]). 
Teорема 3. Пусть $0 \in \rho_{V}\left(\mathfrak{L}_{2}, \mathfrak{L}_{1}\right)$ матрица-функиия

$$
W(\lambda)=\left[\begin{array}{ll}
w_{11}(\lambda) & w_{12}(\lambda) \\
w_{21}(\lambda) & w_{22}(\lambda)
\end{array}\right]
$$

определена равенством (10). Тогда множество матрии рассеяния различных унитарных расширений оператора $V$ описъвается формулой

$$
s(\lambda)=\left(w_{12}(\lambda)+w_{11}(\lambda) \varepsilon(\lambda)\right)\left(w_{22}(\lambda)+w_{21}(\lambda) \varepsilon(\lambda)\right)^{-1}, \quad \lambda \in \rho_{V}\left(\mathfrak{L}_{2}, \mathfrak{L}_{1}\right),
$$

где параметр $\varepsilon(\cdot)$ принадлежит классу Шура $S\left(\mathfrak{N}_{2}, \mathfrak{N}_{1}\right)$ и удовлетворяет условию $0 \in$ $\rho\left(w_{22}(0)+w_{21}(0) \varepsilon(0)\right)$.

сжимающих аналитических оператор-функиий со значениями в $\mathcal{B}\left(\mathfrak{N}_{2}, \mathfrak{N}_{1}\right)$.

Формула (15) следует из аналогичного описания матриц рассеяния для $\widetilde{V}$ :

$$
\widetilde{s}(\lambda)=\left(\widetilde{w}_{12}(\lambda)+\widetilde{w}_{11}(\lambda) \widetilde{\varepsilon}(\lambda)\right)\left(\widetilde{w}_{22}(\lambda)+\widetilde{w}_{21}(\lambda) \widetilde{\varepsilon}(\lambda)\right)^{-1}, \quad \lambda \in \rho_{V}\left(\mathfrak{L}_{2}, \mathfrak{L}_{1}\right),
$$

где параметр $\widetilde{\varepsilon}(\cdot)$ пробегает множество $S\left(\mathfrak{N}_{2}, \mathfrak{N}_{2}\right), \widetilde{w}_{i j}(\cdot)$ - элементы резольвентной матрицы $\widetilde{W}(\cdot)$ для $\widetilde{V}$. При доказательстве формулы (16) используется формула для обобщенных резольвент изометрии $\widetilde{V}$, доказанная в [9]. Именно это обстоятельство и диктует необходимость рассмотрения расширенного оператора $\widetilde{V}$, для которого есть корректное определение дефектного подпространства и спектра, в отличие от оператора $V$.

ЗАмЕчАниЕ 3. В случае гильбертова пространства формула (15) получена в [5]. В [8] эта формула, а также теоремы 1-3 получены методами граничных операторов. В индефинитном случае теория представлений стандартных (т.е. с невырожденным $\operatorname{dom} V$ ) изометрических операторов изучалась в [13], [14]. Отметим, что при нашем подходе удается, во-первых, построить теорию представлений для нестандартных изометрических операторов в $\Pi_{\kappa}$, а во-вторых, упростить некоторые результаты из [13], в частности, формулу (11) за счет иного определения оператор-функций $\mathcal{P}_{\mathfrak{L}_{2}}(\lambda)$ и $\mathcal{Q}_{\mathfrak{L}_{2}}(\lambda)$. Для симметрических операторов соответствующие результаты (теоремы 1-3) получены в [3] и [5].

Автор признателен своему научному руководителю В.А. Деркачу за многочисленные обсуждения и полезные замечания, а также М. М. Маламуду и В.И. Могилевскому за предоставленную рукопись, содержащую доказательства утверждений статьи [7].

\section{СПИСОК ЦИТИРОВАННОЙ ЛИТЕРАТУРЫ}

[1] М. Г. Крейн, Докл. АН СССР, 52 (1946), 651-654. [2] М. Г. Крейн, Ш. Н. Саакян, Докл. АН CСCP, 169 (1966), 1269-1272. [3] V. A. Derkach, M. M. Malamud, J. Funct. Anal., 95:1 (1991), 1-95. [4] V.A. Derkach, J. Math. Sci. (New York), 97:5 (1999), 4420-4460. [5] Д.З. Аров, Л. З. Гроссман, Докл. АН СССР, 270:1 (1983), 17-20. [6] М. М. Маламуд, В.И. Могилевский, Матем. заметки, 73:3 (2003), 460-465. [7] М. М. Маламуд, В. И. Могилевский, Докл. РАН, 395:1 (2004), 11-17. [8] V. E. Katsnelson, A. Ya. Kheifets, P. M. Yuditskii, Topics in Interpolation Theory, Oper. Theory Adv. Appl., 95, Birkhäuser Verlag, Basel, 1997, 283-298. [9] Т. Я. Азизов, И. С. Иохвидов, Основы теории линейных операторов в пространствах с индефинитной метрикой, Наука, М., 1986. [10] Д. В. Байдюк, Укр. матем. вестн., 10:2 (2013), 176-199. [11] Д. В. Байдюк, Тр. Крымской осенней матем. школь, 19 (2009), 1-6. [12] Н. И. Ахиезер, И. М. Глазман, Теория линейных операторов в гилъбертовом пространстве, Т. 1, Вища школа, Харьков, 1977. [13] Г. К. Лангер, Функи. анализ и его прил., 5:4 (1971), 73-75. [14] H. Langer, P. Sorjonen, Ann. Acad. Sci. Fenn. Ser. A I, 561 (1974), 3-45. 